\title{
What is China doing in policy-making to push back the negative aspects of the nutrition transition?
}

\author{
Fengying Zhai ${ }^{1}$, Dawei Fu ${ }^{1}$, Shufa Du ${ }^{1,3}$, Keyou Ge ${ }^{1}$, Chunming Chen ${ }^{2}$ and Barry \\ M Popkin ${ }^{3, *}$ \\ ${ }^{1}$ Institute of Nutrition and Food Hygiene, Chinese Academy of Preventive Medicine, 29 Nan Wei Road, Beijing \\ 100050, People's Republic of China: ${ }^{2}$ Chinese Academy of Preventive Medicine, 27 Nanwei Road, Beijing \\ 100050, People's Republic of China: ${ }^{3}$ Department of Nutrition and Carolina Population Center, CB \# 8120 \\ University Square, University of North Carolina at Chapel Hill, Chapel Hill, NC 27516-3997, USA
}

\begin{abstract}
Objective: To review the nutrition policies and efforts related to nutrition transition in China.

Design and setting: This paper reviews the nutrition policy and activities of China to prevent and control diet-related non-communicable diseases (DR-NCDs). Data came from the Ministry of Health, the Ministry of Agriculture, the State Council and some cross-sectional surveys.

Results: China is undergoing a remarkable, but undesirable, rapid transition towards a stage of the nutrition transition characterised by high rates of DR-NCDs in a very short time. Some public sector Chinese organisations have combined their efforts to create the initial stages of systematic attempts to reduce these problems. These efforts, which focus on both under- and overnutrition, include the new Dietary Guidelines for Chinese Residents and the Chinese Pagoda and The National Plan of Action for Nutrition in China, issued by the highest body of the government, the State Council. There are selected agricultural sector activities that are laudable and few other systematic efforts that are impacting behaviour yet. In the health sector, efforts related to reducing hypertension and diabetes are becoming more widespread, but there is limited work in the nutrition sector. This paper points to some unique strengths from past Chinese efforts and to an agenda for the next several decades.

Conclusions: China is trying in its efforts to prevent and control the development of DR-NCDs but effects are limited. Systematic multi-sector co-operation is needed to effectively prevent and control DR-NCDs inside and outside the health sector.
\end{abstract}

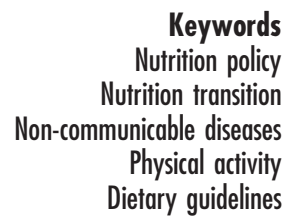

Keywords

Nutrition policy

unicable diseases

Dietary guidelines
China is undergoing a remarkably fast, but undesirable, shift towards a stage of the nutrition transition dominated by a high intake of fat and animal food, as well as a high prevalence of diet-related non-communicable diseases (DR-NCDs) such as obesity, diabetes mellitus, cardiovascular diseases and cancer $^{1,2}$. At the same time, significant pockets of undernutrition and poverty affect many tens of million Chinese ${ }^{1}$. Nevertheless, the present economic cost of DR-NCD equals that for undernutrition, but will be far greater in a few years.

As we show in a separate paper ${ }^{2}$, the shift in the epidemiological transition has occurred, and early death and debility from DR-NCDs are very high in China. Popkin et $a{ }^{1}{ }^{1}$ show that the costs of undernutrition and overnutrition in China were of similar magnitude but the cost ratio of overnutrition to undernutrition is increasing rapidly. They also found that the economic cost of DRNCDs exceeded $2.1 \%$ of the 1995 gross domestic product (GDP) in China ${ }^{1}$.

There are many dimensions to a national policy that attempts to prevent DR-NCDs. Du et $a l^{2}{ }^{2}$ showed that a reduction in energy density and fat intake, and an increase in vegetable intake, are components of a desirable diet strategy. Smoking obviously must be reduced. Moreover, increased moderate- and high-intensity physical activity must be promoted among all ages of the population. Other sectors of the economy must address all of these issues. At the same time, the health sector must take a strong lead, not only to educate the population, but also to improve medical care practice and influence the actions of other government sectors. The efforts from the health sector must produce a strong national nutrition policy that considers the critical nutrition issues, provides guidelines for monitoring dietary intake and nutritional status, and also promotes the type of food guidelines that reduce and prevent DR-NCD.

During the 1990s, it became increasingly clear to the Ministry of Health and key scholars in public health, medicine and nutrition that a remarkably fast shift in the prevalence patterns for DR-NCDs was occurring. Several key changes occurred in national policy documents and the beginnings of systematic efforts to change these 
patterns emerged in the nutrition, health and agriculture sectors. We separate this paper into a discussion of (1) efforts by the nutrition and health sectors; (2) activities of other food sectors, many of which have been instituted by joint nutrition or health efforts with these sectors; and (3) a few pilot education and health promotion activities related to DR-NCD.

\section{Actions in nutrition}

Two major sets of activities led to new national guidelines. One was the development of the Dietary Guidelines for Chinese Residents and the Chinese Pagoda on principles of nutritional science and the present national nutrition situation of China ${ }^{3,4}$. A second was the much broader National Plan of Action for Nutrition. These two activities laid the basis for the initial shifts towards concern about DR-NCDs and related dietary behaviours.

\section{The Dietary Guidelines for Chinese Residents and Chinese Pagoda}

The Chinese Nutrition Society and the leading national organisation, the Institute of Nutrition and Food Hygiene at the Chinese Academy of Preventive Medicine (INFHCAPM), developed eight principles to delineate a good diet, using the Pagoda as a symbol for the same set of good eating principals that guide the United States' dietary pyramid and many similar food-based efforts. These principles, focused on under- and overnutrition and related food behaviours, are as follows.

1. Eat a variety of foods, with cereals as the staple.

2. Consume plenty of vegetables, fruits and tubers.

3. Consume milk, beans or dairy or bean products daily.

4. Consume appropriate amounts of fish, poultry, eggs and lean meat. Reduce fatty meat and animal fat in the diet.

5. Balance food intake with physical activity to maintain a healthy body weight.

6. Choose a light diet that is also low in salt.

7. Drink alcoholic beverages in limited amounts, if at all.

8. Avoid unsanitary and spoiled foods.

A series of smaller activities have been launched to initiate the dissemination of these guidelines. To date, activities have been focused at limited locations and on increased training of public health workers at Antiepidemic Stations throughout the country. For instance, many face-to-face nutrition education sessions have been held in Beijing, Hubei, Shandong, Sichuan and other provinces of China with quite limited attendance to date.

At the same time, professional re-education has begun. As background, beginning in 1949 with the initial Antiepidemic Stations, Chairman Mao proposed the health care strategy of 'yu fang wei zhu' (prevention is the first task of health workers, and health services should be available to residents at all socio-economic levels).
Anti-epidemic Stations at different levels are in charge of collecting information on epidemics and spreading knowledge to prevent and control diseases. The INFH has trained more than 30000 workers and a nutrition network has been established; retraining with the new principles is underway.

A large array of nutrition education publications (e.g. Dietary Guidelines for Chinese Residents, Food Guide Pagoda, How to Get Best Nutrition from Foods) have been created and disseminated in limited, unorganised ways. These publications are in different media (e.g. book, pamphlet, cartoon, movie and decal). However, no systematic national education campaign has been launched.

\section{National Plan of Action for Nutrition}

The State Council of China issued the National Plan of Action for Nutrition in 1997. It was based on many years of discussion and the work of several joint activities of INFHCAPM, the State Council, the Ministry of Health and the Ministry of Agriculture. The general goals are: (1) to ensure an adequate food supply and implement appropriate intervention to alleviate hunger and food shortage; reduce the incidence of energy-protein malnutrition; and prevent, control and eliminate micronutrient deficiencies; and (2) to improve the general nutritional status of the people and prevent DR-NCDs through proper guidance on food consumption behaviour, improvement of dietary patterns and promotion of healthy lifestyles ${ }^{5,6}$.

The plan strengthens intersectoral co-operation and sets targets for agriculture and processed food production. It addresses adjustments to the agriculture structure (e.g. to increase poultry and fishery production; to increase vegetable, fruit and soybean production; and to develop soybean-processing techniques). Some of these targets were fulfilled early while some have not been fulfilled until recently.

\section{Background support}

Several national research and training activities have become important in providing infrastructure and data to support these actions mentioned above. First, a series of national surveys has been developed. These surveys include a national food and nutrition surveillance system, established in 1997, which collected: (1) two rounds of data in 1998 and 2000; (2) three rounds of the China National Nutrition Survey (CNNS) data in 1959, 1982 and 1992; (3) a fourth CNNS round of data funded for 2002; and (4) five rounds of the China Health and Nutrition (CHNS) data in 1989, 1991, 1993, 1997 and 20007,8. These survey data allow the government to understand the nutritional and physical status of the Chinese population, predict trends, and provide a scientific basis for making policy ${ }^{9}$. The second activity providing infrastructure and data is the regional training programme on food and nutrition planning funded by the Netherlands and Chinese 
governments and technically supported by the University of the Philippines. These two actions help to strengthen the national training capabilities by providing nutrition and development workers for the formulation, implementation, monitoring and evaluation of nutrition and related programmes at national, provincial and county levels ${ }^{10}$. A related multi-sector programme focuses on leaders in other sectors (agriculture, economic, education, health, science and technology) of the central and provincial governments. Training has been provided to at least one leader in each of the sectors mentioned above in 12 of 31 Chinese mainland provinces. They are now training food and nutrition workers at county and town levels; trainers and leaders in other provinces will be trained within the next three years.

\section{Related health sector activities}

Systematic national prevention programmes are underway in two areas: hypertension and adult-onset diabetes. A broader National Plan of Prevention and Control of DRNCD was issued in 1996 and integrated intervention activities were carried out in 24 demonstration sites in 17 provinces. These activities included policy reform; supportive environment development (e.g. leadership, institution and human resource development); disease surveillance including mortality, behaviour and socioenvironmental surveillance; and integrated intervention, focused on smoking, non-balanced diet, physical inactivity and the over-intake of salt ${ }^{11}$.

The National Guideline for the Prevention and Control of Hypertension was established in 1998 and included three activities: (1) to measure the blood pressure of patients aged 35 and above during their first clinic visit; (2) to survey the prevalence of hypertension in patients aged 35 and above; and (3) to guide patients with hypertension in controlling blood pressure with diet and medicine. Since 1998 these activities have been conducted at some demonstration cities such as Beijing, Tianjin, Shanghai, Weihai, Liuzhou, Chengdu, Shenyang, Taiyuan, Changsha, Guangzhou and Fuzhou ${ }^{12}$. Other actions have included the designation of October 8th as National Hypertension Day in $1998^{13}$.

The Ministry of Health also issued a national plan to prevent and control diabetes and cardiovascular diseases in 1996. The prevention and control of malignant tumours was listed in the No. 9 Five-Year Development Plan (2000-2005). The government will be giving much more attention to prevent and control DR-NCDs in the future.

\section{Efforts by other sectors}

A series of joint agriculture-nutrition commissions and efforts have been launched, beginning with a joint 1990 international meeting on nutrition and agriculture sponsored by CAPM, INFH, the Ministry of Health and the
Ministry of Agriculture ${ }^{14}$. Several issues were identified at that meeting, including (1) the large decline in soy intake and rapid increase in pork consumption and (2) the low vegetable consumption.

\section{Promotion to develop soybean industry and to increase soybean consumption}

This has been a most important initiative. As various scholars have noted, pork and fat intakes were increasing while soybean intake declined. It is clear that there are important health dimensions to soybeans including known high-quality protein, high dietary fibre and some beneficial flavonoids, as well as some potential as an anticarcinogenic and anti-cardiogenic food. The Ministry of Agriculture has created some initiatives to enhance soy production and consumption in conjunction with the Ministry of Commerce, which is responsible for establishing price controls in the government.

\section{Subsidisation of vegetables}

Vegetable production is very low in northern areas of China, especially in winter. The central government has used price adjustments and subsidies in attempts to increase urban vegetable consumption and the size of these subsidies has grown annually. In addition, there has been a focus on plant breeding to enhance the production of vegetables in the north during the winter. Finally, rural garden promotion activities have been created over the past decade. Researchers have visited many rural regions of the country to disseminate vegetable seeds and introduce methods to plant new kinds of vegetables in home gardens. This effort appears to have increased the production and consumption of vegetables and benefited food markets in both urban and rural areas.

\section{Smoking cessation}

Quite limited efforts are underway to address the growing epidemic of tobacco use in China. Bans were issued on smoking in schools in $1981^{15}$, on tobacco advertisement via the mass media in 1994, on smoking in public places in 1998, and on the sale of cigarettes to adolescents in 2001. Unfortunately, the effects of these bans are few and ineffective.

\section{Physical activity promotion}

The government has attempted to promote physical activity and sports with a 1995 edict $^{16}$. The government calls upon all residents to exercise regularly to improve their physical status and to prevent DR-NCDs; various physical competitions are held at different levels. Increasing numbers of people are now taking part in physical activities.

Most recently, the Ministry of Education created a campaign to lighten students' burdens and to increase time for physical activities; schools are asked to reduce the teaching time and to increase the physical activity time. 
The Ministry of Education is also changing the examination system to decrease the study pressure on students. In the past, university entrance examinations had seven sections; now they have only three, plus one comprehensive test. However, parents of students still want them to do more homework and less physical activity. The effects of the government's efforts are still under observation, but it is felt they are ineffective to date.

\section{Selected intense pilot activities: the Tianjin anti- epidemic station campaign}

A major DR-NCD demonstration project was launched in Tianjin, one of the largest cities in China, in 1989, financed by the World Bank and the World Health Organization (WHO). From 1989 to 1996, a series of measures in health education and health promotion activities were undertaken with a prime focus to reduce hypertension. In 1989 and 1996, two independent samples were drawn from the population in Tianjin demonstration sites for the prevention and control of DR-NCDs to compare the blood pressure levels. The age-adjusted rates of hypertension (systolic pressure $\geq 160 \mathrm{mmHg}$ and/or diastolic pressure $\geq 95 \mathrm{mmHg}$ ) in the 35-64 age group in 1996 (13.44\% in males, $11.43 \%$ in females) are significantly lower than the $17.45 \%$ in males and $15.63 \%$ in females in 1989 , indicating respective rate reductions of $23.0 \%$ and 26.9\%. Similarly, the mortality rate from stroke in the population in the above mentioned demonstration sites was reduced gradually from 160.7 per 100000 in 1985-86 to 83.9 per 100000 in 1994-95. However, the change in the mortality rate from stroke among the whole city population (135.7/100000) was not significant. This indicates that the effect on the control and prevention of stroke in the demonstration sites after implementation of the health education and health promotion strategies and relevant measures was very effective ${ }^{17}$.

Other more limited, smaller efforts among students and other small populations have been undertaken, but no large-scale serious efforts. These limited efforts included the dissemination of dietary guidelines and the conduct of education studies with over 140000 persons in five cities of four provinces. In this effort, an enhanced knowledge of the Chinese Pagoda and the Dietary Guidelines for Chinese Residents was achieved, but there is little evidence of a systematic enhancement of eating behaviour $^{18}$.

\section{Discussion}

Considerations for the role of diet in health and the need to address dietary excess, imbalance and undernutrition have resulted in some very important shifts in the attitudes and understanding of health and nutrition in China. A number of critical activities beginning in the late 1980s through to the 1990s have created some initial effects. The food sector's attempts to enhance soybean and vegetable intake are most prominent; related papers have discussed some issues underlying these changes ${ }^{8,19}$. Most importantly, the Chinese nutrition world has led the way in creating effective multi-sector initiatives, not only with the Ministry of Agriculture and the Ministry of Commerce, but with others. It is clear that a main focus of future work will be the strengthening of these intersectoral collaborations for control and prevention of DR-NCDs in China. Moreover, it is clear that a working group at national level for implementing the survey and surveillance of DRNCDs should be formulated and that a comprehensive intervention strategy should be undertaken for those contributing factors such as hypertension, smoking, unbalanced diet and physical inactivity.

However, more focused activities are still needed within the nutrition sector. One is the need for the nutrition education activities to promote the principles of Dietary Guidelines for Chinese Residents. Another is the need for strengthened guidance on increased physical activity and the dissemination of its benefits to the whole country. Moreover, there is no established dietitian law or governance; this is needed to assist those focused on the restaurant, hotel and hospital food preparers to enhance a healthy diet.

What we have seen are some important, but quite new, efforts to shift the structure of the Chinese diet and to reduce obesity and other poor outcomes and behaviours. More efforts and more intersectoral co-operative efforts are needed to prevent and control the development of DRNCDs.

\section{Acknowledgements}

We thank the Institute of Nutrition and Food Hygiene (INFH), the US National Institutes of Health (NIH/R01-HD30880 and R01-HD38700) and the Fogarty International Center, NIH, for supporting the preparation and publication of this paper. We thank Professors Liming $\mathrm{Li}$ and Gongyuan Yang at the Chinese Academy of Preventive Medicine (CAPM) for providing invaluable information and assistance. We thank Ms Frances Dancy at the University of North Carolina at Chapel Hill for her administrative assistance and Ms Hongfei Hao at INFH for her research assistance.

\section{References}

1 Popkin BM, Horton S, Kim S, Mahal A, Jin S. Diet-related noncommunicable diseases in China and India: the economic costs of the nutrition transition. Unpublished manuscript, 2001.

2 Du S, Lu B, Zhai F, Popkin BM. The nutrition transition in China: a new stage of the Chinese diet. Unpublished manuscript, 2001.

3 The Chinese Nutrition Society. Dietary Guidelines for Chinese Residents and The Food Guide Pagoda. Beijing: People's Medical Publishing House, 1997. 
4 The Chinese Nutrition Society. Dietary guidelines and the food guide pagoda for Chinese residents: balanced diet, rational nutrition and health promotion. Nutr. Today 1999; 34: $106-15$.

5 The State Council. The National Plan of Action for Nutrition in China. Beijing: Beijing University Publishing House, 1997.

6 Ge K, McNutt K. How the Chinese link dietary advice to their national plan of action for nutrition. J. Am. Diet. Assoc. 2000; 100(8): 885-6.

7 Zhai F, Jin S, Ge K. Summary report of China health and nutrition survey (an eight-province case study). J. Hygiene Res. 1996; 25(Suppl.): 16-9.

8 Popkin BM, Ge K, Zhai F, Guo X, Ma H, Zohoori N. The nutrition transition in China: a cross-sectional analysis. Eur. J. Clin. Nutr. 1993; 47: 333-46.

9 Ge K. Summary of the Third National Nutrition Survey. J. Hygiene Res. 1996; 25(Suppl.): 1-8 [in Chinese].

10 Institute of Nutrition and Food Hygiene. Training Program for Food and Nutrition Planning, Beijing, 2001.

11 Ministry of Health. Document of Ministry of Health, Beijing, 1994.

12 Ministry of Health. Document of Ministry of Health, Beijing, 1998 a.
13 Ministry of Health. Document of Ministry of Health, Beijing, 1998b.

14 Ministry of Agriculture. Food and Nutrition in China. Proceedings of a joint meeting organised by the Food and Agriculture Organization (FAO) and sponsored by the Chinese Academy of Preventive Medicine, the Institute of Nutrition and Food Hygiene, the Ministry of Health, and the Academy of Agricultural Science, Beijing, 1990.

15 Ministry of Health. The Collection Documents on Smoking Control in China. Internal document of Ministry of Health, Beijing, 2000.

16 State Administration of Physical Culture and Sports. The Outline for the Sports for All. Document of State Administration of Physical Culture and Sports, China, 1995.

17 Tianjin Municipal Bureau of Health. Report on Tianjin Noncommunicable Disease Community Comprehensive Prevention and Control Project, Tianjin, 1999.

18 Zhao L, Zhai F, Li D, Li Y, et al. A survey on the effects of Dietary Guideline nutrition education project. J. Hygiene Res. 2001; 30: 176-8.

19 Guo X, Popkin BM, Mroz TA, Zhai F. Food price policy can favorably alter macronutrient intake in China. J. Nutr. 1999; 129: $994-1001$. 